\title{
Ocorrência de enteroparasitas na população geronte de Nova Olinda do Norte - Amazonas, Brasil
}

\author{
Ana Felisa HURTADO-GUERRERO ${ }^{1}$; Fernando Hélio ALENCAR²; José Camilo HURTADO-GUERRERO ${ }^{1}$
}

\begin{abstract}
RESUMO
Foi realizado um estudo para avaliar a prevalência de enteroparasitas em um grupo de idosos ribeirinhos, moradores do Município de Nova Olinda do Norte, Estado do Amazonas - Brasil, no período de abril e agosto de 1999. Por meio de um estudo de corte transversal foram analisados 81 exames, através do método de Sedimentação Espontânea (Método de Hoffman et al., 1934). Foi constatada positividade em $72,8 \%$ dos idosos, predominando o monoparasitismo (43,2\%). Os helmintos foram os mais freqüentes (70,4\%), destacando-se: Ascaris lumbricoides (35,2\%), Trichuris trichiura (16,0\%), Ancylostoma duodenale (9,0\%) e Strongyloides stercoralis (9,0\%). Dentre os protozoários (29,5\%), a ocorrência de Entamoeba coli foi de 18,2\%, Giárdia lamblia de 7,0\% e Entamoeba histolytica 4,5\%. Não houve associação estatisticamente significativa entre sexo e grau de parasitismo e entre faixas etárias e condição parasitária. Estes resultados evidenciam um quadro de alta prevalência de parasitas intestinais nesta população e discordam dos reportados por outros pesquisadores quando afirmam que a intensidade da infestação por parasitas diminui na idade avançada. Os achados anteriores exigem das autoridades governamentais medidas de controle e educação para melhorar a qualidade de vida desses idosos, considerando a grave repercussão que esses parasitas tem no estado nutricional dos gerontes de baixa renda.
\end{abstract}

\section{PALAVRAS-CHAVE}

Ocorrência, Enteroparasitoses, Geronte, Ascaris lumbricoides, Amazônia, Nova Olinda do Norte.

\section{Occurrence of enteroparasites in the elderly population of Nova Olinda do Norte, Amazonas, Brazil}

\begin{abstract}
A study was carried out to determine the prevalence of enteroparasites attacking elderly people living in a riverside city of the Municipal district of Nova Olinda do Norte, Amazonas State Brazil. In this cross sectional study were examined the faecal material from 81 elderly people using the method of spontaneous sedimentation (Hoffman et al, 1934). It was found apositiveness in $72,8 \%$ of the elderly people examined, predominating the mono parasitism (43,2\%). The belminths were more frequents (70,3\%): Ascaris lumbricoides (35,2\%), Trichuris trichiura (16,0\%), Ancylostoma duodenale $(9,0 \%)$ and Strongyloides stercoralis $(9,0 \%)$. Among the protozoans (29,7\%) the most frequents were: Entamoeba coli (18,2\%), Giardia lamblia (7,0\%) and Entamoeba histolytica $(4,5 \%)$. \%. There was not a significant statistically association between sex and parasitism degree and between aging profiles and parasitism condition. The above results showed a high prevalence of intestinal parasites in the elderly people of this small town. These results are in disagreement with those reported by another researchers when pointed out that parasitism intensity decreased with aging. The above situation demands from the govern authorities urgent measured of control and education, considering the enormous problems that the enteroparasites cause on the nutritional status and health condition of elderly people with low socio-economic level.
\end{abstract}

KEYWORDS

Helminths, Protozoans, Elderly, Ascaris lumbricoides, Amazon, Nova Olinda do Norte.

${ }^{1}$ CPqL\&MD/FIOCRUZ. Rua Teresina, $n^{\circ}$ 476-Adrianópolis Manaus-AM CEP 69057-070

UEA/ESA, Faculdade de Enfermagem, Av. Carvalho Leal, 1777, Manaus-AM 69065-001.

${ }^{2}$ INPA/CPCS. Caixa Postal 478 Manaus-AM 69011-970 


\section{ACTA \\ AMAZONICA}

OCORRÊNCIA DE ENTEROPARASITAS NA POPULAÇÃO

GERONTE DE NOVA OLINDA DO NORTE - AMAZONAS, BRASIL
As doenças infecciosas e parasitárias ainda constituem uns dos principais problemas de saúde pública, especialmente, nos países em desenvolvimento. Na região Amazônica, por exemplo, os índices de positividade podem alcançar até $95 \%$ em determinados segmentos populacionais, reflexo das precárias condições de vida da referida população (Siqueira \& Castro, 1987; Kilpatrick et al. 1986; Chehter et al. 1994).

Estudos abordando a ocorrência de parasitas intestinais na população idosa são escassos (Araújo \& Correia, 1997), especialmente na Amazônia. Não obstante sabe-se que os enteroparasitas contribuem no comprometimento do estado nutricional deste segmento populacional e da sua saúde como um todo. Esta pesquisa teve como objetivo determinar a ocorrência de enteroparasitas num grupo de gerontes do Município de Nova Olinda do Norte, Amazonas.

Trata-se de um estudo transversal realizado em 1999, que envolveu 81 pessoas idosas ( 44 mulheres e 47 homens), residentes no município de Nova Olinda do Norte, localizado na zona fisiográfica do Amazonas médio, na margem direita do rio Madeira. De cada amostra coletada, foram analisadas três lâminas, através da técnica Qualitativa de Sedimentação Espontânea (método de Hoffman et al. 1934). Confirmado o diagnóstico, a pessoa era encaminhada com um profissional da saúde local para realizar o tratamento. A análise dos dados foi descritiva e inferencial (teste Qui-quadrado), os resultados obtidos com esse teste foram confirmados com Testes Exatos e Permutação de Monte Carlo (Zar, 1996; Manly, 1997). Esta pesquisa contou com a aprovação do Comitê Nacional de Ética de Pesquisa - CONEP (Universidade Federal do Amazonas UFAM).

Os resultados obtidos mostraram um grau de monoparasitismo em $43,2 \%$ dos idosos, seguido de biparasitismo (23,5\%) e poliparasitismo (6,2\%). Para ambos os sexos, constatou-se uma maior ocorrência de monoparasitismo, $47,7 \%$ nas mulheres e $(37,8 \%)$ nos homens (Tabela 1). Não houve associação estatisticamente significativa entre o sexo e o grau do parasitismo $(\mathrm{P}=0,744)$.

A maior freqüência de idosos parasitados foi registrada na faixa de 60 a 69 anos $(83,3 \%)$, entanto que a menor esteve na faixa de 80 a 94 anos (66,7\%) (Tabela 2). Não houve associação entre as faixas etárias e a condição parasitaria $(\mathrm{P}=0,379)$.

O parasita mais comum nos idosos foi $A$. lumbricoides (35,2\%), seguido da E. coli $(18,2 \%)$ e T. trichiura $(16,0 \%)$, e o menos freqüente foi $E$. histolytica (4,5\%). Observou-se que os helmintos $(70,4 \%)$ foram o grupo de parasitas mais predominantes, entanto que os protozoários representaram 29,6\% (Tabela 3).

Estudos como os de Neves et al. (1995) e Ludwig et al. (1999), afirmam que com o avanço dos anos, há uma tendência

Tabela 3 - Freqüência de parasitas intestinais nos idosos de Nova Olinda do Norte - Amazonas, 1999.

\begin{tabular}{llll}
\hline \hline Parasitas Intestinais & $\mathrm{n}$ & $\%$ & $\mathrm{IC} 95 \%$ \\
\hline PROTOZOÁRIOS & & & \\
Entamoeba coli & 16 & 18,2 & 11,$7 ; 30,1$ \\
Giárdia lamblia & 6 & 6,8 & 2,$8 ; 15,4$ \\
$\begin{array}{l}\text { Entamoeba histolytica } \\
\text { HELMINTOS }\end{array}$ & 4 & 4,6 & 1,$4 ; 12,2$ \\
Ascaris lumbricoides & 31 & 35,2 & 27,$7 ; 49,7$ \\
Trichuris trichiuria & 14 & 15,9 & 9,$8 ; 27,3$ \\
Ancilostomídeo & 8 & 9,1 & 4,$4 ; 18,5$ \\
Strongyloides stercoralis & 8 & 9,1 & 4,$4 ; 4,4$ \\
Enterobius vermicularis & 1 & 1,1 & 0,$0 ; 0,1$ \\
Total & 88 & 100 & \\
\hline \hline
\end{tabular}

Tabela 1 - Grau de parasitismo segundo sexo dos idosos em Nova Olinda do Norte - Amazonas, 1999.

\begin{tabular}{ccccccccccc}
\hline \hline \multirow{2}{*}{ Sexo } & \multicolumn{10}{c}{ Grau de Parasitismo } \\
\cline { 2 - 10 } & Não infestados & Monoparasitismo & Biparasitismo & Poliparasitismo & Total \\
\hline Feminino & $\mathbf{n}$ & $\%$ & $\mathbf{n}$ & $\%$ & $\mathbf{n}$ & $\%$ & $\mathbf{n}$ & $\%$ & $\mathbf{n}$ & $\%$ \\
Masculino & 12 & 27,3 & 21 & 47,7 & 9 & 20,5 & 2 & 4,5 & 44 & 100,0 \\
Total & 10 & 27,0 & 14 & 37,8 & 10 & 27,0 & 3 & 8,1 & 37 & 100,0 \\
\hline \hline
\end{tabular}

Tabela 2 - Condição parasitária dos idosos segundo as faixas etárias em Nova Olinda do Norte - Amazonas, 1999.

\begin{tabular}{llllllll}
\hline \hline Faixas etárias & Negativos & \multicolumn{7}{c}{ Positivos } & Total & IC 95\% \\
\cline { 2 - 6 } & $\mathrm{n}$ & $\%$ & $\mathrm{n}$ & $\%$ & $\mathrm{~N}$ & $\%$ & 100,0 \\
\hline 60 a 69 & 4 & 16,7 & 20 & 83,3 & 24 & 62,$6 ; 95,2$ \\
70 a 79 & 12 & 20,8 & 27 & 69,2 & 39 & 100,0 & 52,$4 ; 82,9$ \\
80 a 94 & 6 & 33,3 & 12 & 66,7 & 18 & 100,0 & 41,$0 ; 86,7$ \\
Total & 22 & 27,2 & 59 & 72,8 & 81 & 100,0 & 61,$8 ; 82,1$ \\
\hline \hline
\end{tabular}

$\boldsymbol{x}^{2}=1,94, \mathbf{g l}=2 ; \mathrm{P}=0,379$. IC $95 \%$ = Intervalos de confiança para a proporção de positivos. 


\section{ACTA \\ AMAZONICA}

OCORRÊNCIA DE ENTEROPARASITAS NA POPULAÇÃO

GERONTE DE NOVA OLINDA DO NORTE - AMAZONAS, BRASIL para a diminuição progressiva nas taxas de incidência e de prevalência de enteroparasitas em adultos, principalmente, para A. Lumbricoides, explicada pela mudança de hábitos e desenvolvimento de uma imunidade progressiva de longa duração contra os parasitas. No presente estudo, se evidencia uma situação contraria, pela elevada percentagem de idosos com parasitas intestinais (72,8\%), a qual é comparável com as freqüências encontradas em estudos na região, embora, seja em faixas etárias mais jovens (Souza \& Marinho, 1996; Nagahama et al. 1996; Yuyama et al. 1996; Coura et al. 1993). A. Lumbricoides isoladamente pode desencadear um severo quadro clínico, representado, principalmente, por má digestão, náuseas, perda de apetite, emagrecimento, sensação de irritação nasal, irritabilidade e insônia (Rey, 1992). As famílias de baixa renda e subnutridas são as mais expostas ao risco desta infecção, podendo esta parasita contribuir para debilitar ainda mais seu estado nutricional (Ferreira et al. 2000)

Geralmente, os idosos nestas áreas desenvolvem, no quotidiano, diversas tarefas domesticas, tais como cultivo de hortas caseiras, limpeza do quintal, entre outras, que podem favorecer a contaminação principalmente por geohelmintos, especialmente, em áreas contaminadas por dejetos (Hurtado Guerrero, 2000).

Também é preocupante a ocorrência de T. trichiura, sendo que sua infestação pode ocasionar alterações na motilidade e as funções do intestino grosso (especialmente na reabsorção de líquidos), favorecendo a flatulência e constipação intestinal, um problema de saúde muito comuns em população idosa, agravado pelo baixo consumo de verduras e frutas, principalmente em aqueles que excluem estes itens por apresentarem dificuldade na mastigação (Silva \& Castellanos, 2001).

Na composição deste quadro de enteroparasitas, destacase também a ocorrência dos protozoários, sendo a $E$. coli a mais freqüente. Esta é considerada um indicador de consumo de água e alimentos contaminados por matéria fecal (Knaippe \& Tanus, 1992). Com relação aos protozoários, pesquisas em escolares da periferia de Manaus, evidenciou uma prevalência de $23,9 \%$ de G. lamblia e 18,3\% de E. coli (Souza \& Marinho, 1996) e na região do Nordeste em inquérito coproparasitológico realizado em 365 idosos, se encontrou uma maior prevalência dos protozoários Endolimax nana (33,3\%), E. coli (25,0\%), E. bistolytica (13,9\%) e G. lamblia (2,8\%) (Araújo \& Correia, 1997).

Nesta pesquisa, observou-se que a população idosa apresentou uma maior percentagem de monoparasitismo (43,2\%), enquanto que estudos em crianças e jovens mostraram predominância de poliparasitismo (Araujo \& Correia, 1997; Coura et al., 1993), provalvemente devido a que essas faixas etárias apresentam maior exposição a solos contaminados, especialmente na ausência de calçados (Miranda et al., 1999).

Diversas condições ecológicas favorecem a disseminação dos parasitas intestinais, fazendo com que as helmintoses e protozooses constituam um sério problema de Saúde Pública em algumas regiões de América Latina e do Brasil, podendo alcançar em determinadas regiões um caráter endêmico (Ferreira et al., 2000). Neste sentido, é possível que as altas freqüências de parasitas intestinais registradas neste estudo possam constituir um risco para os idosos com um perfil nutricional comprometido, quadro favorecido por precários hábitos higiênicos, carência de saneamento básico e pela realidade sócio-econômica da região Norte, especialmente, na população idosa ribeirinha (Hurtado Guerrero, 2000).

Estes resultados sugerem uma condição epidemiológica preocupante da população idosa, nesta região, pela alta prevalência de enteroparasitas. Constata-se, ao igual, que essa situação continua, praticamente, inalterada, visto se encontrar nesta pesquisa e em outras realizadas recentemente, percentuais semelhantes aos referidos em estudos realizados na região em outras décadas. Sugere-se, portanto, não somente a realização de outros inquéritos coproparasitológicos direcionados à população geronte na região Amazônica, senão, também, um esforço das autoridades governamentais para implementar medidas preventivas e curativas que permitam a melhoria do nível de vida desses idosos, considerando a grave repercussão que esses parasitas tem no estado nutricional e na saúde dos gerontes de baixa renda.

\section{BIBLIOGRAFIA CITADA}

Araujo, C.F.F.; Correia, J.S. 1997. Freqüência de parasitas intestinais em idosos dos núcleos da Prefeitura de João Pessoa, Estado da Paraíba. RBAC, 29(4): 230-231.

Coura, J.R.; Willcox, H.P.F.; Tavares, A.M.; Paiva, D.D.; Fernandes, O.; Rada, E.L.J.; Perez, E.P.; Borges, L.C.L.; Hidalgo, M.E.C.; Nogueira, M.L.C. 1993. Aspectos epidemiológicos, sociais e sanitários em áreas do meio de Solimões I: Estudo nas localidades de São Francisco do Laranjal, Aranaí e são Lázaro do Surubim, Município de Coari, Amazonas. Anais da Academia Nacional de Medicina, 153 (3):122-126.

Chehter, L.; Cabeça, M.; Catapani, W.R. 1994. Parasitoses intestinais. Rev. Bras. Med., 51: 125-132.

Ferreira, M.U.; Ferreira, C.S.; Monteiro, C.A. 2000. Tendência secular das parasitoses intestinais na infância na cidade de São Paulo (1984-1996). Rev. Saúde Pública, 34(6): 73-82. $<$ http://www.scielosp.org/scielo.php

Hurtado Guerrero, A.F. 2000. Fatores determinantes do estado nutricional do idoso de Nova Olinda do Norte-Amazonas. Dissertação de Mestrado, Universidade do Amazonas/ Instituto Nacional de Pesquisa da Amazônia. Manaus, Amazonas. 123pp.

Hoffmann, W.A.; Pons, J.A.; Janer, J.L. 1934. Sedimentation concentration method in schistosomiasis, Puerto Rico. J. Public Health, 9: 283-298.

Knaippe, F.; Tanus, R. 1992. Prevalência de giardíase e flutuação sazonal em uma amostra da população urbana da região centro-oeste do Brasil. Rev. Bras. Farm., 73(2): 33-34.

Kilpatrick, M.E.; Escamilla, J.; Townsend, A.B.; López, W.G.; Vargas, E.P.; Castillo, L.B. 1986. Parasitosis intestinales identificadas mediante examen de heces en tres grupos de población del Peru. Bol. Of. Sanit. Panam., 100(4): 412415 . 
Ludwig, K.M.; Frei, F.; Alvares Filho, F; Ribeiro-Paes, J.T. 1999. Correlação entre condições de saneamento básico e parasitoses intestinais na população de Assis, Estado de São Paulo. Rev. Soc. Bras. Med. Trop., 32(5): 547-555. < http:/ /www.scielo.br/scielo.php.

Manly, B. F. J. 1997. Randomization, Bootstrap and Monte Carlo Methods in Biology. $2^{\text {nd }}$ Ed. Chapman and Hall, London. 399pp

Miranda, R.A.; Xavier, F.B.; Nascimento, L.R.J.; Menezes, R.C., 1999. Prevalência de parasitismo intestinal nas aldeias indígenas da tribo Tembé, Amazônia Oriental Brasileira. Rev. Soc. Bras. Med. Trop., 32(4): 1-8.

Nagahama, D.; Yuyama, L.K.O.; Cortez, M.H.A.; Alencar, F.H.; Fisberg, M. 1996. Perfil alimentar e ocorrência de infestação parasitária em pre-escolares de creches municipais da cidade de Manaus - Am. In SBAN - V Congresso Nacional da Sociedade Brasileira de Alimentação e Nutrição. São Paulo, p.180, SP-148.

Neves, D.P.; Melo, A.L.; Genaro, O.; Linardi, P.M. 1995. Parasitologia Humana, 9a edição. Atheneu. São Paulo, $524 \mathrm{pp}$

Rey, L. 1992. Bases de Parasitologia Médica. Guanabara Koogan. Rio de Janeiro, 349pp
Siqueira, R.V.; Castro, O.J. 1987. Incidência de parasitoses intestinais no Município de Alfenas - MG: principais fatores que contribuiriam para a disseminação. Rev. Esc. Farm. Odont., 11.

Silva, S.R.C.; Castellanos, R.A.F. 2001. Autopercepção das condições de saúde bucal por idosos. Rev. Saúde Pública, 35(4):349-355. <http://www.scielosp.org/scielo.php

Souza, T.P.; Marinho, H.A. 1996. Avaliação nutricional em escolares de $1^{\mathrm{a}}$ a $4^{\mathrm{a}}$ série na periferia de (zona leste) da cidade de Manaus - Am. In SBAN - IV Congresso Nacional da Sociedade Brasileira de Alimentação e Nutrição. São Paulo, p17.

Yuyama, L.K.O.; Aguiar, J.P.L.; Nagahama, D.; Alencar, F.H.1996. Avaliação da dieta dos pré-escolares do município de Itapiranga - Calha do rio Amazonas. In: $S B A N$ - V Congresso Nacional da Sociedade Brasileira de Alimentação e Nutrição. Temas Livres - Saúde Pública, São Paulo, p127.

Zar, J.H. 1996. Biostatistical Analysis. Third Edition. Prentice Hall International Editions. USA. 662pp + Appendices.

RECEBIDO EM 19/02/2002

ACEITO EM 03/11/2005 